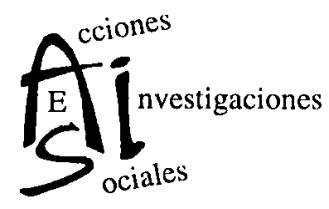

\title{
El Consejo Aragonés del Cooperativismo Participación del Movimiento Cooperativo en la vida económica y social Aragonesa
}

\author{
Ángel GutiérRez Díez
}

PROFESOR DEL DIPLOMA DE ESPECIALIZACIÓN EN DIRECCIÓN DE ORGANIZACIONES DE ECONOMÍA SOCIAL

Se analiza el Decreto regulador del Consejo Aragonés del Cooperativismo, publicado recientemente. Se comienza exponiendo las circunstancias que han dado lugar a su aparición y el proceso de elaboración de la norma. A continuación se entra en su estudio, incidiendo especialmente en el alcance real de las funciones que tiene encomendadas y el campo de maniobra que resulta.Se sigue con el análisis de su composición y funcionamiento, deteniéndose en la forma en que se configura el necesario equilibro que ha de darse entre los representantes de la Administración y del Movimiento Cooperativo, así como de los medios que se ponen a su servicio. En las conclusiones, se lleva a cabo una valoración acerca de su futuro y de las posibilidades que se abren con su puesta en marcha.

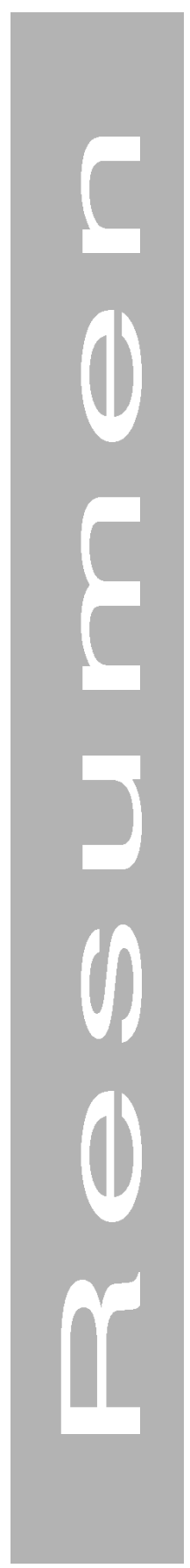




\section{El Consejo Aragonés del Copera- tivismo. Participación del Movimiento}

Cooperativo en la vida económica Ángel Guitierez Díez y social Aragonesa

\section{Introducción, elaboración de su Reglamento y naturaleza}

La Disposición Adicional $4^{\text {a }}$ de la Ley 9/1998, de Cooperativas de Aragón, crea el Consejo Aragonés del Cooperativismo como órgano consultivo del Gobierno de Aragón, para cumplir adecuadamente los fines de promoción y desarrollo cooperativos que tiene encomendados. Su composición será mixta, integrando tanto a representantes de las organizaciones de cooperativas constituídas conforme a la Ley Aragonesa, como a representantes de la Administración Autonómica. Al mismo tiempo, la regulación de su organización y competencias específicas se remite a desarrollo reglamentario posterior. El Decreto 65/2.003, de 8 de abril, del Gobierno de Aragón, publicado en el B.O.A. de 5-5-2.003, por el que se regula el Consejo Aragonés del Cooperativismo, viene a dar cumplimiento a este mandato legal y constituye su Reglamento.

Como cuestión preliminar, interesa resaltar que la Ley obliga a que la elaboración de esta norma reguladora se realice previa consulta a las organizaciones representativas del Cooperativismo. A tal efecto, en la elaboración del proyecto de Decreto se dio audiencia a las organizaciones representativas del sector, en concreto a las tres Federaciones Aragonesas que integran, respectivamente, el Cooperativismo Agrario, de Trabajo Asociado y de Viviendas, que presentaron las alegaciones correspondientes. Así mismo, el texto fue informado por los Servicios Jurídicos de la Administración de la Comunidad Autónoma y por el resto de Departamentos del Gobierno de Aragón que, por razón de las ma- 
terias de su competencia, inciden de forma directa en el mundo empresarial y social cooperativo, como Agricultura o Vivienda, entre otros. De esta manera, el borrador previamente propuesto por el Órgano competente en materia de Registro de Cooperativas del Departamento de Economía, Hacienda y Empleo, resultó notablemente enriquecido con las propuestas aportadas.

En cuanto a su naturaleza jurídica, el Consejo queda adscrito orgánicamente al Departamento competente en materia de Cooperativas, sin que se le atribuya personalidad jurídica propia.

\section{Funciones de Consejo}

Las funciones del Consejo Aragonés del Cooperativismo se recogen en el artículo 3 de su Decreto regulador. A este respecto, hay que señalar que no estamos ante una enumeración de carácter cerrado, sino que el último punto del precepto abre la posibilidad de encomendar al Consejo en el futuro cualquier otra función que se le pueda asignar en razón de su naturaleza.

En cuanto a los cometidos que se enuncian, los tres primeros se refieren a la facultad de participar, como órgano consultivo, en la elaboración de normas. En primer lugar, se prevé de forma explícita que el Consejo informará y elaborará dictámenes acerca de la disposiciones normativas que se emitan por el Gobierno de Aragón, que afecten a uno o varios de los sectores del Cooperativismo Aragonés. En el mismo sentido, el punto primero del artículo 99 de la ley de Cooperativas de Aragón, señala también que la Diputación General de Aragón consultará con las Federaciones y Confederaciones de Cooperativas competentes por razón de la materia, los proyectos de disposiciones de carácter general que les afecten.

Estamos por tanto ante una obligación de consulta, siempre que la materia a regular afecte de forma directa a un sector cooperativo. Se podría afirmar incluso que el pronunciamiento que lleve a cabo el Consejo sobre una cuestión determinada, sería independiente y compatible con el que pueda efectuar sobre el mismo asunto una determinada entidad asociativa de Cooperativas de un sector, al ser aquel un órgano representativo del Cooperativismo en General. Así mismo se puede requerir 
también, de forma facultativa para el Gobierno de Aragón, que el Consejo se pronuncie sobre cualquier otra materia que estime conveniente, aunque no tenga una vinculación directa con el sector cooperativo.

Esta última previsión resulta de extraordinario interés y plantea un debate en torno a la cuestión de hasta donde debe llevar el Gobierno Aragonés la participación de las entidades representativas del Sector Cooperativo, y por extensión de la Economía Social, en los distintos órganos consultivos existentes en la Comunidad. El mismo punto uno del artículo 99 de la Ley Aragonesa dispone que la Diputación General de Aragón instrumentará la participación del Movimiento Cooperativo en las instituciones y órganos públicos bajo su dependencia, en los consejos asesores de la Administración Autonómica, así como en las decisiones que adopte cada uno de los Departamentos en las materias de la respectiva competencia.Este ultimo apartado, puesto en relación con la declaración explícita de reconocimiento de la importancia del Movimiento Cooperativo dentro del sistema económico de la Comunidad Autónoma, que se plasma en el artículo 97 de la Ley, abre la puerta a una participación plena de este sector, a todos los niveles, en los órganos consultivos y participativos de la Comunidad.

Este debate también pone sobre la mesa otro tema. La Ley de Cooperativas de Aragón regula las Cooperativas y el Movimiento Cooperativo, pero el concepto de Economía Social, en función de la amplitud que demos a sus límites, puede agrupar realidades y sectores mucho más amplios, algunos de reciente aparición y otros que son tanto o más antiguos que las propias Cooperativas. El abanico podría incluir desde las Asociaciones y Fundaciones con participación directa en el entramado socio-económico de nuestro entorno, hasta las Sociedades Laborales, en las que si bien su punto de origen se halla en el Derecho Mercantil, su ley reguladora las configura como entidades en las que el capital y la toma de decisiones estará siempre del lado de los socios que obtienen sus medios de subsistencia a partir del trabajo en la empresa que constituye el objeto de la sociedad. La Economía Social en su conjunto, podría muy bien tener voz propia a través de su agrupación en un órgano consultivo específico, o mediante su participación en las máximas instancias consultivas de la Comunidad Autónoma, junto con otros sectores que ya la tienen. 
La misma función de carácter consultivo que la Ley y el Reglamento regulador otorga al Consejo Aragonés del Cooperativismo, se recoge en todas las leyes y reglamentos autonómicos como la primera competencia de los órganos similares que regulan.En Aragón, como en otras Comunidades, a esta función le acompañan otras que vienen a dar mayor contenido a esta figura.

En esta línea, el punto 4 del artículo tres del Decreto regulador, encomienda al Consejo la función de participar en la promoción y difusión del Cooperativismo y sus principios en el ámbito de la Comunidad, a través de las actividades formativas o de cualquier otra naturaleza, así como en el fomento de las relaciones intercooperativas. Este apartado viene a dar cumplimiento directo a la declaración de intenciones que en el mismo sentido se recoge en el artículo 97 de la Ley Aragonesa.En este precepto la Diputación General de Aragón, en función de sus competencias, declara expresamente de interés social la promoción, estímulo y desarrollo de las Cooperativas y de sus organizaciones representativas dentro de su territorio, y en base a ello adoptará en sus programas de actuación las medidas necesarias para promover la constitución y el desarrollo de cooperativas y el mejor cumplimiento de sus objetivos.

El Consejo Aragonés del Cooperativismo se configura por tanto como órgano de participación e instrumento de la Administración Aragonesa para la promoción y difusión del Cooperativismo. En consecuencia, esta función requiere una participación activa del propio Consejo en las medidas de esta naturaleza que lleve a cabo la Comunidad Autónoma.Debe destacarse de forma especial la mención expresa a las actividades de carácter formativo, dado que la formación es uno de los principios básicos del movimiento cooperativo y por ello ha de ser la principal formula para su promoción y estímulo. En base a esta previsión normativa, se reserva al Consejo un papel de actor principal en todas aquellas actuaciones de carácter formativo en el ámbito del sector cooperativo, que sean financiadas o promovidas desde la Administración Autonómica.

Se asigna también al Consejo el fomento de las relaciones intercooperativas. Hay que tener en cuenta que la gran variedad de actividades de carácter económico que se agrupan bajo el paraguas de la forma jurídica Cooperativa, que incluso se orga- 
nizan a través de distintas organizaciones sectoriales, precisan de una adecuada coordinación. A este respecto, debe hacerse constar que el artículo 93,5 de la Ley de Cooperativas de Aragón configura la "Confederación de Cooperativas de Aragón" como ente destinado a aglutinar el movimiento asociativo de este carácter en nuestro territorio. Tampoco debe dejarse de lado la tarea que en este campo desarrollan las distintas Uniones y Federaciones sectoriales. Por tanto, la actividad del Consejo en esta materia ha de llevarse a cabo en colaboración con las citadas entidades.

La tareas de promoción y fomento del desarrollo y de la educación cooperativa, y la de facilitar la colaboración entre las entidades y empresas que se agrupan bajo sus principios, son otras de las que aparecen encomendadas de forma general a los distintos consejos del cooperativismo en las Comunidades Autónomas que los regulan. En este sentido, hay que tener en cuenta que el Movimiento Cooperativo, por su propia naturaleza, trasciende las fronteras de cualquier territorio y fija como uno de sus objetivos principales la intercooperación a todos los niveles. El Consejo Aragonés del Cooperativismo está llamado por tanto a desempeñar un papel importante en este campo, en representación de nuestra Comunidad.

La siguiente función del Consejo también tiene acogida en las distintas regulaciones españolas de consejos autonómicos, aunque con desigual nivel de implicación. Se trata de la colaboración con la Administración Autonómica en los expedientes de Descalificación, Intervención temporal, Disolución y Liquidación de Cooperativas, para los que sea requerido por aquella.

Entrando en el análisis del precepto, como primer matiz destaca que corresponde a la Administración la facultad de determinar en que procesos ha de actuar. En segundo lugar, parece desprenderse de la redacción del precepto, que si el órgano correspondiente del Gobierno de Aragón solicita la colaboración del Consejo, éste ha de prestarla necesariamente. Por otro lado, el Reglamento no determina en qué forma y con qué alcance se prestará la colaboración. Estas cuestiones y otras derivadas, como la necesidad de que el Consejo pueda tener a su disposición personal con la preparación suficiente en caso de que se le requiera un apoyo de carácter técnico, habrán de ser dilucidadas en el futuro. 
Los procesos de disolución y liquidación aparecen regulados en los artículos 67 a 69 de la Ley de Cooperativas de Aragón. En primer lugar, la colaboración puede prestarse a través del asesoramiento o informe en determinados casos de disoluciones y liquidaciones conflictivas. Aunque no aparece contemplado específicamente en el Reglamento del Consejo, también cabría la posibilidad de colaboración con los Órganos Judiciales, en aquellos casos en que la designación de liquidadores deba efectuarse por el Juez. Así mismo, los remanentes de las liquidaciones de Cooperativas, que han de ser entregados a sus entidades asociativas y que durante un año al menos han de conservarse depositados y con posterioridad destinarse a fines de promoción y fomento cooperativo, podrían ser objeto de gestión por parte del Consejo. Por último, como cuestión importante queda la de los procesos de liquidación de oficio previstos en la disposición transitoria segunda de la Ley, para aquellos casos en que la Cooperativa no se haya adaptado en tiempo y forma a la misma. En este tema el Consejo podría jugar un papel interesante.

En lo que se refiere a la Intervención temporal de Cooperativas, el Consejo será probablemente la entidad más adecuada para proponer al Gobierno de la Comunidad Autónoma el nombramiento de los Interventores previstos en el artículo 96,2 de la Ley de Cooperativas de Aragón, a través del Consejero competente en la materia. Por último, en lo relativo a su participación en los expedientes de descalificación de Cooperativas, previstos en el artículo 95 de la Ley, además de su intervención en los procesos de disolución y liquidación iniciados a consecuencia de la misma, sería conveniente solicitar informe del Consejo durante la tramitación del expediente, aunque no esté previsto expresamente por dicha norma.

La siguiente función del Consejo consiste en prestar asesoramiento, a requerimiento de las partes, en los conflictos que se susciten entre las Cooperativas, entre ellas y sus socios, o en el seno de las Cooperativas entre estos últimos, limitado este supuesto a aquellos casos en que la cuestión litigiosa se derive de materias propias de la competencia de la Cooperativa. Se establece además que puede contemplarse de forma expresa esta posibilidad en los Estatutos Sociales o en el Reglamento de Régimen Interno de cada Cooperativa. 
Hay que señalar en primer lugar, que el precepto que regula este tema evita la mención directa de las figuras de la conciliación o arbitraje cooperativos, que se prevén de forma explícita en alguna otra normativa autonómica, aunque por ello tampoco ha de entenderse que por principio hayan de quedar excluidas. Por tanto, se deja a voluntad de las partes y todo lo más a lo previsto en las normas internas de cada Cooperativa, la utilización y el alcance de esta posibilidad de mediación. En segundo lugar, está la cuestión relativa a la capacidad y disposición del Consejo para prestar esta asistencia. Para llevar a cabo una actuación profesional y de carácter amplio, es necesario disponer también del personal técnico adecuado que pueda desempeñar esta tarea de forma eficaz. De la forma en que se articulen estas cuestiones en el futuro, dependerá el gran medida el papel real que ha de jugar el Consejo en este tema.

Los apartados 7, 8 y 9 del artículo tres del Decreto regulador del Consejo, le encomiendan funciones de seguimiento del correcto cumplimiento por las Cooperativas de su normativa y principios reguladores. El séptimo le asigna en concreto la función de colaborar con los órganos de control y seguimiento de la Administración de la Comunidad Autónoma, a quienes competa velar por la correcta aplicación y cumplimiento de la normativa que afecte de forma directa a cualquiera de los sectores del Cooperativismo en Aragón. La forma en que ha de llevarse a cabo este cometido no aparece concretada, por lo que todo dependerá de cómo se desarrolle esta cuestión en el futuro. La fiscalización y seguimiento del correcto cumplimiento por las Cooperativas Aragonesas de lo dispuesto en su Ley reguladora, se encomienda en el artículo 94 de la misma al Departamento de Sanidad, Bienestar y Trabajo -hoy al Departamento de Economía, Hacienda y Empleo-, a través de la Inspección de Trabajo y Seguridad Social, sin perjuicio de las competencias que otros órganos tengan atribuidas en la materia. Por tanto, la colaboración puede circunscribirse únicamente al ámbito del Departamento que resulta directamente competente o, por el contrario y así sería deseable, extenderse a todos los que de at guna u otra forma inciden en la aplicación de la normativa que afecta al sector cooperativo.

Los puntos 8 y 9 del Reglamento, asignan al Consejo las funciones de proponer actuaciones para impulsar el efectivo cum- 
plimiento de los principios cooperativos y la gestión democrática y participativa en el seno de las Cooperativas y del movimiento de las representa, y velar por la correcta utilización de los fondos de educación y promoción. Se trata en realidad de una extensión de las funciones de control previstas en el apartado anterior, que en este caso le son encomendadas de forma directa.

\section{Composición y funcionamiento del Consejo}

El Reglamento regulador del Consejo prevé que su composición será mixta, integrando tanto a representantes del Gobierno de Aragón, como de las Entidades Asociativas de Cooperativas. Se aparta nuestra norma del criterio seguido en otros consejos autonómicos, que prevén la integración en su seno de expertos cooperativos y de representantes de otras instancias, como pueden ser la Universidad, el Parlamento Autonómico o la Administración Local.

El equilibrio que ha de existir en su configuración lo resuelve el Reglamento estableciendo una composición de carácter paritario. De los dieciséis miembros que lo integran, la Presidencia se otorga al Consejero competente en materia de Cooperativas. Los siete miembros restantes en representación de la Administración Autonómica han de ser Directores Generales con competencia en las materias que aparecen enumeradas en el artículo 4 del Decreto, que corresponden a los sectores económicos en que actualmente posee mayor incidencia el Cooperativismo.En lo que se refiere a los ocho representantes del Movimiento Cooperativo, se determina que se asignarán dos miembros a cada una de las Federaciones Aragonesas de Cooperativas de Trabajo Asociado y de Cooperativas Agrarias, y otro a la Federación que agrupe a las de Viviendas. En lo que se refiere a los tres miembros restantes, se atribuirán por el Presidente del Consejo a representantes de las Entidades Asociativas de Cooperativas previstas en el artículo 93 de la Ley Aragonesa, atendiendo a criterios de representatividad del sector cooperativo.Se deja así la puerta abierta al otorgamiento de representación a cualquier otra entidad asociativa que pudiera constituirse en el seno de alguno de los sectores cooperativos que aún no han creado una estructura organizativa. Así mismo, cada miembro del Consejo habrá de designar un suplente, para el caso de imposibilidad de asistencia.Los cargos durarán cuatro años. 
La Vicepresidencia corresponderá a un representante de las Entidades Asociativas de Cooperativas, elegido por el pleno del Consejo, el cual designará también al Secretario. En este último caso se prevén dos posibilidades: la primera es elegir a un miembro del propio Consejo para ejercer este cometido, y la otra designar a un funcionario de la Administración de la Comunidad Autónoma cuyas funciones tengan relación con la materia de Cooperativas, asistiendo en este caso con voz y sin voto a sus reuniones.

El funcionamiento del Consejo se configura en base a los caracteres generales previstos en la normativa aragonesa para los órganos colegiados, pudiendo funcionar en Pleno, en Comisión Permanente 0 en Comisiones de Trabajo. En este aspecto, las regulaciones de las distintas normativas autonómicas configuran un esquema muy parecido, que es seguido por el Reglamento Aragonés.

\section{Financiación y medios a su servicio}

Es ésta una de las cuestiones más delicadas al abordar el estudio del Reglamento regulador del Consejo.Su artículo 13 determina que la financiación se hará con cargo a las partidas de gastos del Presupuesto de la Comunidad Autónoma, teniendo los créditos previstos con tal finalidad carácter ampliable, a fin de poder generar crédito a su vez las cantidades que pudieran transferirse o donarse para el cumplimiento de sus fines propios. El presupuesto de la Comunidad no ha previsto todavía ninguna partida específica para el Consejo, aunque sí existe alguna línea destinada a subvencionar acciones de fomento y promoción de la Economía Social, cuyos fondos podrían utilizarse en principio para su financiación.Como ingresos de carácter extraordinario, se contemplan únicamente cantidades transferidas o donadas por terceros. Quizás hubiese sido interesante haber dejado abierta la puerta a otras posibilidades, como son las cantidades procedentes de remanentes de liquidación de Sociedades Cooperativas.

Una cuestión paralela que puede plantearse en este apartado es la posibilidad y conveniencia de que el Consejo pueda tener una cuenta independiente para la administración de sus fondos. Se trata de un órgano para el que no se ha previsto la 
atribución de personalidad jurídica propia, por lo que en principio puede ser difícil su materialización.

El último tema a tratar es la omisión que existe en el Decreto regulador de los medios materiales y personales que puedan adscribirse al Consejo. Si bien la sede se fija en la ciudad de Zaragoza, no se le transfiere ninguna dependencia destinada a llevar a cabo sus cometidos, ni se prevé la existencia de personal a su servicio.

\section{Conclusiones}

Nos encontramos ante una figura jurídica novedosa en Derecho Aragonés, que comenzará su andadura una vez se convoque su sesión constituyente a instancias del Consejero de Economía, Hacienda y Empleo del Gobierno de Aragón. Se trata de un ente necesario, que vendrá a articular la colaboración entre la Administración Pública de nuestra Comunidad y el Movimiento Cooperativo.Su Decreto regulador, que posteriormente ha de ser completado por disposiciones de régimen interno que regulen el funcionamiento de sus órganos, resulta en principio suficiente para su puesta en marcha, atribuyéndole funciones de notable importancia en aras a la promoción del Cooperativismo y, por extensión, de la Economía Social en general.

En cuanto a su desarrollo futuro, el factor determinante será la voluntad y el trabajo de las partes que lo integran. El Consejo puede permanecer como ente cuya función principal y casi única sea la meramente consultiva, o puede jugar en cambio un papel más activo, interviniendo directamente en la gestión del mundo cooperativo, a través de la colaboración con la Administración en cuestiones como la liquidación de Cooperativas, su intervención temporal o, entre otras, la puesta en marcha de mecanismos de solución de conflictos que surjan en el mundo cooperativo. Si su papel acaba siendo el de órgano imprescindible y activo en relación con las funciones que tiene encomendadas, en el futuro gozará probablemente de un marco normativo de actuación más holgado, que le otorgue mayor independencia y adscripción de medios. 